one-dose. Vaccinated cohorts that include incompletely vaccinated individuals offer the opportunity to evaluate the effectiveness of reduced dosing schedules. We aimed to estimate effectiveness of one-dose of quadrivalent vaccine against highgrade squamous intraepithelial lesion (HSIL) and cervical intraepithelial neoplasia grade 2 or higher (CIN2+).

Methods Data-linkage was performed between the populationbased Cervical Cancer Screening Program and immunization registries in BC. Occurrence of HSIL and CIN2+ were compared in a screening cohort of YW born between 1994-2005 who were either (a) unvaccinated; (b) completely vaccinated per-schedule (2-doses 150 days apart or 3-doses) between 914 years of age; or (c) vaccinated between 9-14 years of age with one-dose. Relative incidence rates (RR, (95\%CI)) were calculated using Poisson regression and adjusted for birth year and age at first screening.

Results Overall, 19,496 women were unvaccinated, 14,130 were completely vaccinated (mean age at vaccination 13.3 \pm 1.2 ), and 471 vaccinated with one dose only (mean age at vaccination $13.4 \pm 1.1$ ). We found significant protection among completely vaccinated compared to unvaccinated women. The adjusted RR for HSIL was $0.52(0.43-0.64)$ and for CIN2+ $0.42(0.31-0.57)$. No significant protection after one dose against HSIL and CIN2 + was observed compared with unvaccinated women, respective adjusted RR 0.69 (0.27-1.41) and $1.21(0.43-2.86)$.

Conclusion In this observational study, no evidence of protection of one-dose against HSIL and CIN2+ was observed, while protection was found amongst completely vaccinated. The small sample size and the potential for administrative data biases may have impacted this preliminary analysis. This methodological approach provides a platform for further analyses, with larger numbers, to determine the potential impact of single dose HPV vaccination.

Disclosure No significant relationships.

\section{P833 HSV-2 SEROSTATUS AND HPV INCIDENCE, PERSISTENCE, AND PRECANCEROUS LESIONS IN A COHORT OF HPV-VACCINATED WOMEN LIVING WITH HIV}

${ }^{1}$ Elisabeth Mcclymont* ${ }^{2}$ François Coutlée, ${ }^{3}$ Marette Lee, ${ }^{4}$ Arianne Albert, ${ }^{5}$ Sharon Walmsley, ${ }^{4}$ Nancy Lipsky, ${ }^{6}$ Gina Ogilvie, ${ }^{7}$ Darrell Tan, ${ }^{6}$ Deborah Money. ${ }^{7}$ University of British Columbia, Obstetrics and Gynecology, Vancouver, Canada; '2l'Université de Montréal, Microbiologie Médicale et Infectiologie, Montreal, Canada; ${ }^{3} B C$ Cancer, Vancouver, Canada; ${ }^{4}$ Women's Health Research Institute, Vancouver, Canada; ${ }^{5}$ University Health Network, Toronto, Canada; ${ }^{6}$ University of British Columbia, Vancouver, Canada; ${ }^{7}$ St. Michael's Hospital, Toronto, Canada

10.1136/sextrans-2019-sti.878

Background In understanding HPV oncogenesis, several co-factors have been proposed including co-infection with HSV-2. We assessed the relationship between HSV-2 serostatus and HPV-related outcomes in a cohort of quadrivalent HPV-vaccinated women living with HIV (WLWH).

Methods In this multi-site study of immunogenicity and efficacy of the qHPV vaccine in WLWH, three doses of $\mathrm{qHPV}$ vaccine were offered. Visits were at months $-3,0,2,6,12$, 18, 24, and annually thereafter. Participants provided clinical data and cervico-vaginal swabs for HPV DNA detection (Linear array assay) at each visit; baseline serum was tested for HSV-2 type-specific serology (Focus EIA). We used non-parametric statistics to compare the HPV-related outcomes (including 37 high and low-risk HPV types) according to HSV-2 serostatus.

Results 151 women aged $\geq 16$ provided baseline serum samples for HSV-2 testing. The predominant regions of origin were Canada (51\%) and Africa (30\%). At baseline, median age was 39 years (IQR: 34-45), median CD4 count was 500 cells $/ \mathrm{mm}^{3}$ (IQR: 382-692), and 70\% had an HIV viral load $<50$ copies/mL. Baseline seroprevalence of HSV-2 was 76.2\%, and median years of follow-up was similar for HSV-2 positive (6, IQR: 5.0-7.8) and negative (6, IQR: 5.2-7.9) participants. HSV-2 positivity was significantly associated with increased age. HSV-2 seropositive and seronegative participants had similar frequencies of HPV persistence $(86 / 115$ vs $27 / 36, p=1)$, clearance of incident HPV infections (88/115 vs 26/36, $\mathrm{p}=0.8)$, number of HPV types detected during the study $(4.5$ vs $5.7, \mathrm{p}=0.1)$, HSIL cytology during the study (11/115 vs $2 /$ $36, \mathrm{p}=0.7)$, and CIN2+ histology ever $(15 / 115$ vs $5 / 36$, $\mathrm{p}=1)$. Results were similar in sensitivity analyses in which HSV-2 seropositivity was defined as an index value $\geq 3.5$.

Conclusion HSV-2 seropositivity was common in this cohort of WLWH in Canada, but was not associated with multiple measures of HPV incidence, persistence, and precancerous lesions.

Disclosure No significant relationships.

\section{P834 EPIDEMIOLOGY OF HUMAN PAPILLOMAVIRUS AMONG WOMEN IN GUANGDONG, CHINA 2008-2017}

${ }^{1}$ Ganfeng Luo*, ${ }^{2}$ Xiaoli Sun, ${ }^{1}$ Meijuan Li, ${ }^{2}$ Xiping Luo, ${ }^{1}$ Huachun Zou. ${ }^{1}$ Sun Yat-Sen University, School of Public Health (Shenzhen), Guangzhou, China; ${ }^{2}$ Guangdong Women and Children Hospital, Department of Gynecology, Guangzhou, China

\subsection{6/sextrans-2019-sti.879}

Background Human papillomavirus (HPV) infection is the major etiological factor for cervical cancer. Data on the prevalence and genotype distribution of HPV infection in Guangdong Province in southern China are limited. Our study aimed to analyze genotype-specific, age-specific prevalence and year-on-year trend of HPV detection among women in Guangdong 2008-2017.

Methods Exfoliated cervical cells were harvested from women attending gynecological department or medical examination center at Guangdong Women and Children Hospital. Twentyone HPV subtypes were tested. Chi-squared test and the linear-by-linear association test were applied.

Results A total of 199,963 women attending gynecological department and 11,999 women attending medical examination center were included. HPV was detected in $20.16 \%$ of gynecological outpatients, with $17.67 \%$ positive for high risk (HR) HPV and $4.43 \%$ positive for low risk (LR) HPV. HPV was detected in $17.25 \%$ of women receiving physical examinations, with the prevalence of HR HPV and LR HPV being $14.88 \%$ and $4.05 \%$, respectively. HPV prevalence significantly differed among these two populations $(\mathrm{P}<0.001)$. The five most prevalent genotypes were HPV 52, 16, 58, 81 and 53 among gynecological outpatients and 52, 81, 58, 53 and 16 among women receiving physical examinations. The distribution of any HPV, HR HPV, LR HPV and 9-valent HPV infections showed a bimodal pattern across age groups among both populations. A quasi-V-shaped prevalence curve was observed over the 10-year period among gynecological outpatients for any HPV, HR HPV subtype, LR HPV subtype and 9-valent HPV, while a quasi-reversed $\mathrm{V}$-shaped curve was observed among 